\title{
REVIEW: AN ENHANCEMENT OF ROAD SCENES CAPTURED IMAGES USING HDCP \& COLOR DISCRIPTOR
}

\author{
Lakhveer kaur ${ }^{1 *}$, Rajbhupinder kaur $^{2}$ \\ ${ }^{* 1,2}$ Department of Computer Engineering, Punjabi University Yadavindra College of Engineering, Talwandi Sabo \\ Punjab, India \\ *1 Email: lakhveerdhaliwal49@gmail.com
}

*Corresponding Author: -

Email ID-lakhveerdhaliwal49@gmail.com

\begin{abstract}
: -
The visibility of images of outdoor road scenes will generally become degraded when captured during inclement weather conditions. Drivers often turn on the headlights of their vehicles and streetlights are often activated, resulting in localized light sources in images capturing road scenes in these conditions. Additionally, sandstorms are also weather events that are commonly encountered when driving in some regions. A novel and effective haze removal approach to remedy problems caused by localized light sources and color shifts, which thereby achieves superior restoration results for single hazy images. The Road image degradation can cause problems for intelligent transportation systems such as traveling vehicle data recorders and traffic surveillance systems, which must operate under a wide range of weather conditions. The objective of this work is to implement the Road Scenes Captured by Intelligent Transportation Systems using Hybrid technique. To enhance the images using different filters and enhancement techniques.
\end{abstract}

Keywords: - PSNR, MD and Processing Speeds.

\section{(c) (\$) (i)}




\section{INTRODUCTION}

Visibility in road images can be degraded due to natural atmospheric phenomena such as haze, fog, and sandstorms. This visibility degradation is due to the absorption and scattering of light by atmospheric particles. Road image degradation can cause problems for intelligent transportation systems such as traveling vehicle data recorders and traffic surveillance systems, which must operate under a wide range of weather conditions [1]. The amount of absorption and scattering depends on the depth of the scene between a traffic camera and a scene point; therefore, scene depth information is important for recovering scene radiance in images of hazy environments. Traffic

Scene classification is an emerging topic with considerable importance in the field of intelligent transportation systems. With the increased availability of cameras in vehicles (either on mobile devices or as embedded hardware in luxurious car models), there are more and more possibilities for simplifying common intelligent transportation tasks. We are especially interested in improving fleet management systems. Fleet management systems are used to track the status of fleets of vehicles belonging to various kinds of companies (e.g. taxi, delivery, cargo transport etc.). They use GPS sensors to track the location of the vehicle, but have little information about the vehicle's environment. Some useful information about the vehicle's surroundings can be inferred by using a camera to record images from the driver's perspective, and then solving a classification problem to detect interesting types of traffic scenes and scenarios. For example, this approach can be used to identify traffic jams, or to differentiate open road environments from urban/rural roads or tunnels. [2] Image classification in general is a common topic in computer vision, extensively researched in great number of papers. Active research focuses mainly on recognizing images in a large number of diverse classes [1]. The performance of new image classification techniques is usually evaluated on one or more of many publicly available benchmark datasets. This enables a simple and meaningful comparison of state-of-the-art methods applied on various domains. However, the scene radiance recovered via the dark-channel-prior-based techniques is usually accompanied by the generation of serious artifacts when the captured hazy road image contains localized light sources or color-shift problems due to sandstorm conditions. This can be problematic for many common road scenarios. For example, in inclement weather conditions, the drivers generally turn on headlights when they are driving in order to improve visual perception, and streetlamps are lit for similar reasons. The techniques based on the dark channel prior cannot produce satisfactory restoration results when presented with these situations. A novel haze removal approach by which to avoid the generation of serious artifacts by the conjunctive utilization of the proposed hybrid dark channel prior (HDCP) module, the proposed color analysis (CA) module, and the proposed visibility recovery (VR) module. The proposed technique can effectively conceal localized light sources and restrain the formation of color shifts when the captured road image contains localized light sources or color-shift problems.

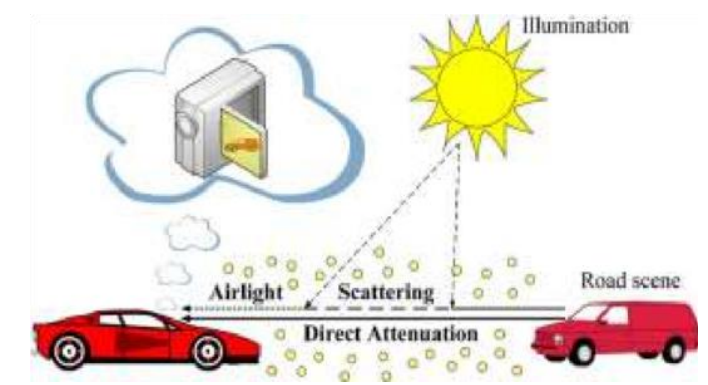

Fig.1. Pictorial description of hazy image acquisition via the optical model.

\section{a) Optical Model}

In computer vision and pattern analysis, the optical model is widely used to describe the digital camera information of a hazy image under realistic atmospheric conditions in the $R G B$ color space as $I^{c}(x, y)=J^{c}(x, y) t(x, y)+A^{c}(1-t(x$, y) $) \ldots \ldots . .1$

where $c \in\{r, g, b\}, I^{c}(x, y)$ represents the captured image, $J^{c}(x, y)$ represents the scene radiance that is the ideal haze free image, $A c$ represents the atmospheric light, and $t(x, y)$ represents the transmission map describing the portion of the light that arrives at a digital camera without scattering. The first term of (1), i.e., $J^{c}(\mathrm{x}, \mathrm{y}) \mathrm{t}(\mathrm{x}, \mathrm{y})$ represents the direct attenuation describing the decayed scene radiance in the medium. The second term of (1), i.e., $A^{c}(1-t(x, y))$, represents the air light.

\section{b) HDCP Module}

The dark channel prior technique [3]s can work well for haze removal in single images that lack localized light sources. However, haze removal by the dark channel prior technique [2] usually results in a seriously underexposed image when the captured scene features localized light sources. The proposed HDCP module can produce a restored image that is not underexposed by using a procedure based on the dark channel prior technique [3]. The dark channel prior technique in [1] can employ large patch size operation for the captured image in order to acquire the correct atmospheric light. However, the use of a large local patch will result in invariable transmission and thereby leads to the generation of halo effects in the recovered image. In contrast, when the dark channel prior technique [1] uses a small patch size, the recovered image will not exhibit halo effects.

However, localized light will be misjudged as atmospheric light. Hence, we present the HDCP module that ensures correct atmospheric light estimation and the subsequent avoidance of halo effects during the haze removal of single images based on the hybrid dark channel prior technique. This technique will be introduced in the following. To 
effectively estimate the density of the haze featured by an image, we combine the advantages of small and large patch sizes via different weights. In addition, we use the large patch size to acquire the correct atmospheric light during the implementation of the hybrid dark channel prior technique.

\section{RELATED WORK}

Previous Research work on the road scene image enhancement is done by different researchers. The different research views of different researchers are given below:

Shih-Chia Huang et al. [2014] have presented the visibility of images of outdoor road scenes will generally become degraded when captured during sinclement weather conditions. Drivers often turn on the headlights of their vehicles and streetlights are often activated, resulting in localized light sources in images capturing road scenes in these conditions. Additionally, sandstorms are also weather events that are commonly encountered when driving in some regions. In sandstorms, atmospheric sand has a propensity to irregularly absorb specific portions of a spectrum, thereby causing color-shift problems in the captured image. Traditional state-of-the-art restoration techniques are unable to effectively cope with these hazy road images that feature localized light sources or color-shift problems. In response, we present a novel and effective haze removal approach to remedy problems caused by localized light sources and color shifts, which thereby achieves superior restoration results for single hazy images. The performance of the proposed method has been proven through quantitative and qualitative evaluations. [1].

Ivan et al. [2014] have studied work deals with multi-label classification of traffic scene images. We introduce a novel labeling scheme for the traffic scene dataset FM2. Each image in the dataset is assigned up to five labels: settlement, road, tunnel, traffic and overpass. They propose representing the images with (i) bag-of-words and (ii) GIST descriptors. The bag-of-words model detects SIFT features in training images, clusters them to form visual words, and then represents each image as a histogram of visual words. On the other hand, the GIST descriptor represents an image by capturing perceptual features meaningful to a human observer, such as naturalness, openness, roughness, etc. We compare the two representations by measuring classification performance of Support Vector Machine and Random Forest classifiers. Labels are assigned by applying binary one-vs-all classifiers trained separately for each class. Categorization success is evaluated over multiple labels using a variety of parameters. They report good classification results for easier class labels (road, F1 $=98 \%$ and tunnel, F1 =94\%), and discuss weaker results (overpass, F1 $<50 \%$ ) that call for use of more advanced methods. [2].

Bo Li et al. [2013] have presented as a challenging problem, image haze removal plays an important role in computer vision applications. The dark channel prior has been widely studied for haze removal since it is simple and effective; however, it still suffers from oversaturation, artifacts and dark-look. To resolve these problems, this study proposes a method of single image haze removal using content-adaptive dark channel and post enhancement. The main contributions of this work are as follows: first, an associative filter, which can transfer the structures of a reference image and the grey levels of a coarse image to the filtering output, is employed to compute the dark channel efficiently and effectively. Secondly, the dark channel confidence is utilised to restrict the dark channel based on the content of the image. Finally, a post enhancement method is devised to map the luminance of the restored haze-free image with the preservation of local contrast. Experimental results demonstrate that the proposed method significantly improves the visibility of the hazy image.[5].

Ajay Raghavan et al. [2012] have presented unattended camera devices are increasingly being used in various intelligent transportation systems (ITS) for applications such as surveillance, toll collection, and photo enforcement. In these fielded systems, a variety of factors can cause camera obstructions and persistent view changes that may adversely affect their performance. Examples include camera misalignment, intentional blockage resulting from vandalism, and natural elements causing obstruction, such as foliage growing into the scene and ice forming on the porthole. In addition, other persistent view changes resulting from new scene elements of interest being captured, such as stalled cars, suspicious packages, etc. might warrant alarms. Since these systems are often unattended, it is often important to automatically detect such incidents early.[3].

Kaiming He et al. [2011] have presented dark channel prior to remove haze from a single input image. The dark channel prior is a kind of statistics of outdoor haze-free images. It is based on a key observation-most local patches in outdoor hazefree images contain some pixels whose intensity is very low in at least one color channel. Using this prior with the haze imaging model, we can directly estimate the thickness of the haze and recover a high-quality haze-free image. Results on a variety of hazy images demonstrate the power of the proposed prior. Moreover, a high-quality depth map can also be obtained as a byproduct of haze removal.[6].

Fan-Chieh Cheng et al. [2011] have proposed a novel background subtraction approach in order to accurately detect moving objects. The method involves three important proposed modules: a block alarm module, a background modeling module, and an object extraction module. The block alarm module efficiently checks each block for the presence of either a moving object or background information. This is accomplished by using temporal differencing pixels of the Laplacian distribution model and allows the subsequent background modeling module to process only those blocks that were found to contain background pixels. Next, the background modeling module is employed in order to generate a highquality 
adaptive background model using a unique two-stage training procedure and a novel mechanism for recognizing changes in illumination. The overall results show that our proposed method attains a substantially higher degree of efficacy, outperforming other state-ofthe-art methods by Similarity and $F 1$ accuracy rates of up to $35.50 \%$ and $26.09 \%$, respectively. [4].

KokKeong Tan et al. [2000] have presented the degradation of images by the atmosphere often restricts imaging applications to good visibility conditions. For example, when imaging the terrain from a forward-looking airborne camera, the atmospheric degradation causes both a loss in contrast and color information. Enhancement of such images is a difficult task due to the complexity in restoring both the luminance and chrominance while maintaining go One particular problem is the fact that the level of contrast loss depends strongly on wavelength; shorter wavelengths i.e. blue are more effected. In this paper, a novel method is presented for the enhancement of color images. This method is based on the underlying physics of the degradation and the parameters required for enhancement. The proposed method is tested using synthetic images to explore the limitations and reliability of the method under different visibility conditions. [7].

\section{Why to Study this work?}

A novel and effective haze removal approach to remedy problems caused by localized light sources and color shifts, which thereby achieves superior restoration results for single hazy images. The Road image degradation can cause problems for intelligent transportation systems such as traveling vehicle data recorders and traffic surveillance systems, which must operate under a wide range of weather conditions. Another problem is that the captured hazy road image contains localized light sources or color-shift problems due to sandstorm conditions. Motion detection is known to be one of the greatest problem areas. There is darkness problem in the road scene images due to sandstorms.

\section{Comparison Table of the Work}

\section{CONCLUSION}

\begin{tabular}{|c|c|c|c|}
\hline $\begin{array}{l}\text { Author } \\
\text { Name }\end{array}$ & $\begin{array}{l}\text { Year of } \\
\text { Publicati } \\
\text { on }\end{array}$ & $\begin{array}{l}\text { Technique } \\
\text { Used }\end{array}$ & Result \\
\hline $\begin{array}{l}\text { ShihChia } \\
\text { Huang }\end{array}$ & 2014 & Restoration & $\begin{array}{l}\text { To evaluate } \\
\text { the } \\
\text { performanc } \\
\text { e of Haze } \\
\text { removel }\end{array}$ \\
\hline \multirow[t]{2}{*}{ Ivan } & 2014 & SIFT & $\begin{array}{l}\text { To remove } \\
\text { the Haze } \\
\text { with two }\end{array}$ \\
\hline & & & $\begin{array}{l}\text { factors } \\
\text { percentage } \\
\text { F1=98\%an d } \\
\text { F1=945 }\end{array}$ \\
\hline Bo Li & 2013 & $\begin{array}{l}\text { adaptive ark } \\
\text { channel and } \\
\text { post } \\
\text { enhancement }\end{array}$ & $\begin{array}{l}\text { Enhanced } \\
\text { the image } \\
\text { with the } \\
\text { help of } \\
\text { luminance }\end{array}$ \\
\hline $\begin{array}{l}\text { Ajay } \\
\text { Raghav } \\
\text { an }\end{array}$ & 2012 & $\begin{array}{l}\text { intelligent } \\
\text { transportatio } n \\
\text { systems }\end{array}$ & $\begin{array}{l}\text { To enhance } \\
\text { image based } \\
\text { on } \\
\text { color }\end{array}$ \\
\hline $\begin{array}{l}\text { Kaimin g } \\
\text { He }\end{array}$ & 2011 & $\begin{array}{l}\text { dark channel } \\
\text { prior }\end{array}$ & $\begin{array}{l}\text { Enhance and } \\
\text { recover the } \\
\text { high } \\
\text { e removal } \\
\text { image }\end{array}$ \\
\hline
\end{tabular}

The research work is to enhance the road scene images using HDCP, color model and morphological operations. In this work the different researchers studied different techniques to enhance the images, but there are different problems that I have studied in this work. In the future work the research work is implemented with the help of HDCP and pixel descriptor and any other techniques.

\section{References}

[1].Huang, C.H., Chen, B.H., and Cheng, Y.J., (2014), “An Efficient Visibility Enhancement Algorithm for Road Scenes Captured by Intelligent Transportation Systems", IEEE Transactions on Intelligent Transportation Systems, Vol. 15, No. 5 . 
[2]..Brkic, K., Horvatin, I., and Segvic, S., (2014), "Multi-Label Classification of Traffic Scenes", Proceedings of the Croatian Computer Vision Workshop.

[3].Raghavan, A., Price, R. and Liu, J., (2012), "Detection of Scene Obstructions and Persistent View Changes in Transportation Camera Systems",2012 15th International IEEE Conference on Intelligent Transportation Systems Anchorage, Alaska, USA.

[4].4 .Cheng, F.C., and Ruan, S.J., (2012), “ Accurate Motion Detection Using a Self-Adaptive Background Matching Framework", IEEE Transactions On Intelligent Transportation Systems, Vol. 13, No. 2.

[5].Li, B., Wang, S., Zheng, J., and Zheng, L.,(2013), " Single image haze removal using content-adaptive dark channel and post enhancement:, IET Comput. Vis, Vol. 8, Iss. 2, pp. 131-140.

[6].He, K., Sun, J., and Tang, X., (2011), “Single Image Haze Removal Using Dark Channel Prior”, IEEE transactions on pattern analysis and machine intelligence, vol. 33, no. 12.

[7].Tan, K., and Oakley, J.P., (2000), "Enhancement of color images in poor visibility conditions," in Proc. ICIP, vol. 2, pp. 788-791.

[8].Narasimhan,G., and S. Nayar, K.,(2003), “Contrast restoration of weather degraded images,” IEEE Trans. Pattern Anal. Mach. Intell., vol. 25, no. 6, pp. 713-724.

[9].Li, W. J., Gu,B., Huang, J.T., Wang, S.Y., and Wang, M.H.,(2012), "Single image visibility enhancement in gradient domain," IET Image Process., vol. 6, no. 5, pp. 589

[10]. Doshi, A., and Adrian, G., (2010), “ Optical Flow Diffusion with Robustified Kernels ” Image Vis. Computs., vol. 28 , no. 12 , pp. $1575-1589$.

[11]. Wang, W.J., Chen, B.H., and Huang, S.C.,(2013), "A novel visibility restoration algorithm for single hazy images," in Proc. IEEE Int. Conf. Syst., Man, pp. 847-851. 